\title{
Application of a cervical low incision in the functional neck dissection of thyroid papillary carcinoma
}

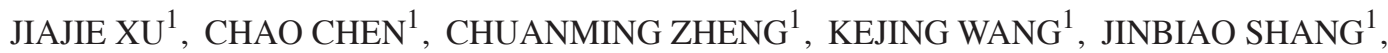 \\ XIANHUA FANG ${ }^{2}$, MINGHUA GE ${ }^{1 *}$ and ZHUO TAN ${ }^{1 *}$
}

Departments of ${ }^{1} \mathrm{Head}$ and Neck Surgery, and ${ }^{2}$ Pathology, Zhejiang Cancer Hospital, Hangzhou, Zhejiang 310022, P.R. China

Received June 16, 2015; Accepted November 11, 2015

DOI: $10.3892 / \mathrm{mco} .2016 .745$

\begin{abstract}
The present study aimed to discuss the advantage of the application of a cervical low incision for functional neck dissection in patients with thyroid papillary carcinoma. The study was a retrospective analysis of 87 thyroid papillary carcinoma patients; cervical low incision in the functional neck dissection was applied for 47 cases and the classic ' $L$ ' incision was applied for 40 cases. The different integrity, surgical time, blood loss and the aesthetic property of the incision were compared between the cervical low incision and the classic ' $L$ ' incision for lateral neck dissection of thyroid cancer. The postoperative pathological diagnosis was that the average total amount and the region II lymph nodes of the unilateral neck dissection were 33 and 10 for the cervical low incision group, and 32 and 11 for the classic ' $L$ ' incision group, respectively $(\mathrm{P}>0.05)$. The average unilateral neck dissection times were 87 and 58 min for the cervical low incision group and the classic ' $\mathrm{L}$ ' incision group, respectively $(\mathrm{P}<0.05)$. The blood loss of the cervical low incision group was $67 \mathrm{ml}$, while the loss for the classic ' $L$ ' incision group was $61 \mathrm{ml}(\mathrm{P}>0.05)$. The postoperative incision of the cervical low incision group was smaller and more concealing. Additionally, the cosmetic deformities were milder for an inconspicuous cervical scar, and the sensation was improved for the patients in comparison with the classic ' $L$ ' incision group. These results suggest that the application of cervical low incision for functional neck dissection in thyroid papillary carcinoma patients aids in reducing postoperative complications, without increasing recurrence rates. Therefore, the classic ' $\mathrm{L}$ ' incision can be replaced by the cervical low incision.
\end{abstract}

Correspondence to: Dr Zhuo Tan, Department of Head and Neck Surgery, Zhejiang Cancer Hospital, 38 Guangji Road, Hangzhou, Zhejiang 310022, P.R. China

E-mail: 03kqyxxjj@163.com

*Contributed equally

Key words: classic 'L' incision, cervical low incision, neck dissection, thyroid papillary carcinoma, eight reserved

\section{Introduction}

Thyroid papillary carcinoma is a type of malignant cancer that has a low malignancy tendency and a long survival time. Although the rate of metastasis of the cervical lymph node is high, $40-70 \%$ (1), the therapeutic effect is good. Currently, the general treatment for cervical lymph node metastasis of the papillary thyroid cancer is neck lymph node dissection $(2,3)$. Although there is significant controversy regarding the application of the technique, it still has a crucial role in the surgical therapy of thyroid cancer and the main type is the ' $\mathrm{L}$ ' incision (4). For good exposure and convenience of surgery, ' $\mathrm{L}$ ' incision is used in the majority of surgeons' cases. However, a scar is evident on vertical incision. Such scar tissue following surgery affects the appearance of the patients. As removing a section of the supraclavicular cutaneous nerve would cause postoperative sensory loss to the neck and ear lobe area (5), and consequentially reduce the patients' quality of life, the low neck incision and 'eight reserved' neck dissection (vena jugularis interna, vena jugularis externa, sternocleidomastoid, accessory nerve, superclavicular nerve, transverse cervical artery, musculus omohyoideus and auricular nerve were reserved) were performed to reduce the side effects of the neck dissection to an optimal level.

\section{Materials and methods}

General materials. The surgical and pathological prospective protocols of a series of patients with a histological diagnosis of papillary thyroid cancer between July 2004 and September 2006 were reviewed. Patients were excluded from the study if they: i) Had a history of previous thyroid surgery or ii) were referred for completion thyroidectomy or for recurrent cancer (local or nodal metastases). This third criterion was operated in order to avoid attributing to the thyroid procedure, the sequelae of the parathyroid surgery. Based on these criteria, the study population comprised 87 patients. These 87 papillary thyroid cancer patients were treated in Zhejiang Cancer Hospital (Zhejiang, China). The patients include 21 males and 66 females. The male to female ratio was 1:3.14. The age range of the patients was from 12 to 67 years old, and the median was 35 years old. The patients had been confirmed to exhibit papillary thyroid cancer by intra-operative frozen pathology or were diagnosed postoperatively as papillary cancer. 
Table I. Patient demographics.

\begin{tabular}{lccc}
\hline Demographics & Total no. of cases $(\mathrm{n}=87)$ & Cervical low incision $(\mathrm{n}=47)$ & Classic ' $\mathrm{L}$ ' incision $(\mathrm{n}=40)$ \\
\hline Male, $\mathrm{n}(\%)$ & $21(24.1)$ & $11(23.4)$ & $10(25.0)$ \\
Female, $\mathrm{n}(\%)$ & $66(75.9)$ & $36(76.6)$ & $30(75.0)$ \\
Mean age, years (range) & $35(12-67)$ & $36(12-67)$ & $33(12-65)$ \\
$\mathrm{T}_{1} \mathrm{~N}_{0} \mathrm{M}_{0}, \mathrm{n}(\%)$ & $22(25.3)$ & $9(19.1)$ & $13(32.5)$ \\
$\mathrm{T}_{1} \mathrm{~N}_{1} \mathrm{M}_{0}, \mathrm{n}(\%)$ & $60(69.0)$ & $35(74.5)$ & $25(62.5)$ \\
$\mathrm{T}_{1} \mathrm{~N}_{2} \mathrm{M}_{0}, \mathrm{n}(\%)$ & $5(5.7)$ & $3(6.4)$ & $2(5.0)$ \\
\hline
\end{tabular}

Incision. While performing cervical low incision (supracervical arc incision), eight functional tissues were conserved for the patients. These were the vena jugularis interna, vena jugularis externa, sternocleidomastoid, accessory nerve, superclavicular nerve, transverse cervical artery, musculus omohyoideus and auricular nerve. However, the classic ' $\mathrm{L}$ ' incision only reserves the sternocleidomastoid, vena jugularis interna and accessory nerve.

Surgical procedures. While performing cervical low incision, a supracervical arc incision on the lesion side was first executed (usually $2 \mathrm{~cm}$ above the collarbone) (Fig. 1). The posterior edge of this incision reached the anterior edge of the trapezius muscle so that the incision could extend to provide a good exposure subsequent to the creation of the flap. For the patients with a long neck, sometimes the incision is lengthened a little backwards. Subsequently, the sternocleidomastoid, vena jugularis interna and vena jugularis externa were dissociated to protect the auricular nerve, and the lymph nodes along the vena jugularis interna were dissected (Fig. 2). Following this, the neck dissection was performed and the nerve at cervical levels II and III was dissociated. Finally, all branches of the cutaneous nerve were dissociated and preserved from the top to bottom. The range of neck dissection was levels II-VI. The commonly employed classic ' $L$ ' incision for thyroid cancer is a continuation of a Kocher incision along the posterior border of the sternocleidomastoid muscle superiorly to approximately 1 inch below the ipsilateral ear lobe (4). These procedures were performed as described previously (4). The range of conventional neck dissection was II-VI level lymph nodes and provided good exposure.

Follow-up. All the patients were followed up to 31-60 months, with an average of 41 months. The tumor extent was defined according to the 7th edition of the American Joint Committee on cancer (AJCC) tumor-node-metastasis (TNM) classification of malignant tumors in 2010. Quantitative data are expressed as the mean \pm standard deviation; qualitative data are expressed as percentages. $\mathrm{P}<0.05$ was considered to indicate a statistically significant difference.

\section{Results}

Patient demographics and types of surgery. Patient demographics are shown in Table I. Table II lists the types of surgical procedures. Among the 87 patients, 3 had partial thyroidectomy in local hospitals; however, postoperation pathology confirmed these cases as papillary thyroid cancer. The other
Table II. Types of surgery.

\begin{tabular}{lccc}
\hline Surgery & $\begin{array}{c}\text { Total no. } \\
\text { of cases }(\%)\end{array}$ & $\begin{array}{c}\text { Cervical low } \\
\text { incision }\end{array}$ & $\begin{array}{c}\text { Classic ' } L \text { ' } \\
\text { incision }\end{array}$ \\
\hline Total thyroidectomy & 87 & 47 & 40 \\
Unilateral dissection & $83(95.4)$ & $45(95.7)$ & $38(95.0)$ \\
Bilateral dissection & $4(4.6)$ & $2(4.3)$ & $2(5.0)$ \\
\hline
\end{tabular}

84 patients received their initial treatment at Zhejiang Cancer Hospital. Intraoperative frozen pathology confirmed those cases as thyroid papillary carcinoma. More specifically, 5 of the 84 initial treatment patients were found to have enlarged cervical lymph node or metastatic lymph node by preoperative fine needle aspiration. A total of 47 cases of patients, including 11 males $(23.4 \%)$ and 36 females $(76.6 \%)$, were treated by the cervical low incision and 40 cases, including 10 males $(25.0 \%)$ and 30 females $(75.0 \%)$, were treated by the classic ' $\mathrm{L}$ ' incision. According to the 2002 AJCC staging of thyroid papillary carcinoma, 22 cases were $\mathrm{T}_{1} \mathrm{~N}_{0} \mathrm{M}_{0}$. A total of 60 cases were $\mathrm{T}_{1} \mathrm{~N}_{1} \mathrm{M}_{0}$ and 5 were $\mathrm{T}_{1} \mathrm{~N}_{2} \mathrm{M}_{0}$. In the cervical low incision group, 9 patients $(19.1 \%)$ were at $\mathrm{T}_{1} \mathrm{~N}_{0} \mathrm{M}_{0}$ stage, 35 (74.5\%) were $\mathrm{T}_{1} \mathrm{~N}_{1} \mathrm{M}_{0}$ and $3(6.4 \%)$ were $\mathrm{T}_{1} \mathrm{~N}_{2} \mathrm{M}_{0}$. In the classic ' $\mathrm{L}$ ' incision group, 13 patients $(32.5 \%)$ were at $\mathrm{T}_{1} \mathrm{~N}_{0} \mathrm{M}_{0}$ stage, $25(62.5 \%)$ were $\mathrm{T}_{1} \mathrm{~N}_{1} \mathrm{M}_{0}$ and $2(5.0 \%)$ were $\mathrm{T}_{1} \mathrm{~N}_{2} \mathrm{M}_{0}$ (Table I). Among the 87 patients, 4 exhibited bilateral papillary thyroid carcinoma (implemented bilateral neck dissection) and 83 had unilateral papillary thyroid cancer. Bilateral dissection was performed in 2 cases $(4.3 \%)$ of the cervical low incision group and in 2 cases $(5 \%)$ of the classic ' $L$ ' incision group. Additionally, unilateral dissection was performed in 45 cases $(95.7 \%)$ of the cervical low incision group and in 38 cases $(95.0 \%)$ of the classic ' $L$ ' incision group (Table II).

Lateral neck dissection time. The surgical time ranged from 50-190 min in the cervical low incision group and 30-75 min in the classic ' $\mathrm{L}$ ' incision group, with an average of 87 and 58 min, respectively. $(\mathrm{P}<0.05)$ (Fig. $3 \mathrm{~A}$ and $\mathrm{B})$. The dissection time of the cervical low incision group was longer than that of the classic ' $\mathrm{L}$ ' incision group.

Lymph node number during neck dissection. For the cervical low incision group there were 16 to 49 lymph nodes on each side and 18 to 50 lymph nodes for the classic ' $\mathrm{L}$ ' incision group. For the former, the average total amount and the region II 


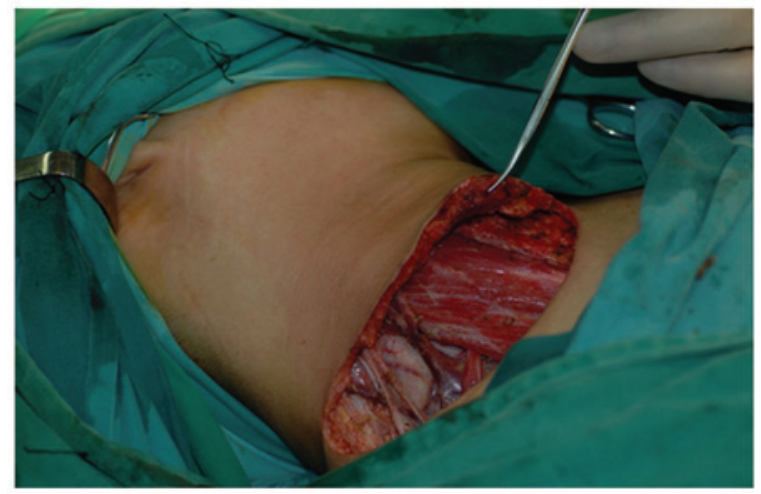

Figure 1. Supracervical arc incision on the lesion side.

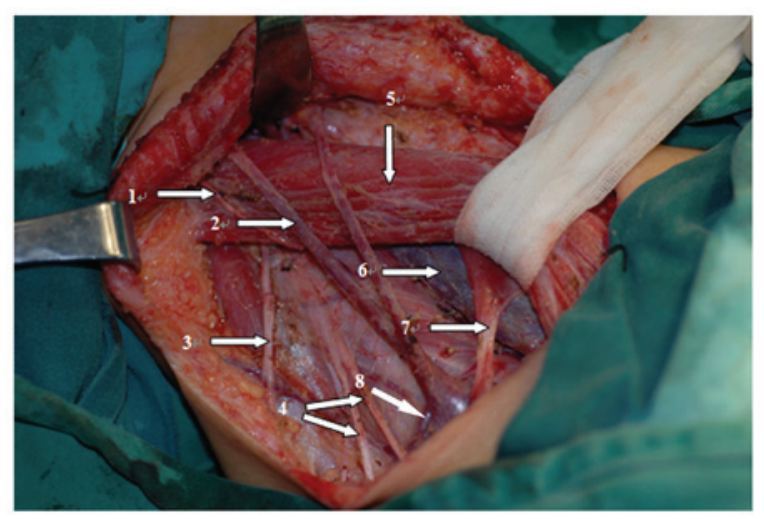

Figure 2. Reservation of normal structures. 1., Auricular nerve; 2., vena jugularis externa; 3., accessory nerve; 4., supraclavicular nerve; 5., sternocleidomastoid; 6., vena jugularis interna; 7., musculus omohyoideus; 8. , transverse cervical artery.

lymph nodes of the unilateral neck dissection were 33 and 10 , and for the latter they were 35 and 11 , respectively $(\mathrm{P}>0.05)$ (Fig. 4A and B). In total, cervical lymph node metastasis was pathologically confirmed in 35 patients ( 37 sides) of the cervical low incision group and 30 (32 sides) in the classic ' $L$ ' incision group. On average, 4.8 and 4.2 metastasis nodes were identified on each side for the two groups, respectively (Table III).

Blood loss during neck dissection. Blood loss in the cervical low incision group was 50-100 $\mathrm{ml}$ on each side with an average of $67 \mathrm{ml}$, while for the classic ' $\mathrm{L}$ ' incision group it was $40-80 \mathrm{ml}$ with an average of $61 \mathrm{ml}(\mathrm{P}>0.05)$ (Fig. 5A and B).

Complication ratings. No fatalities were recorded. Two patients with cervical low incision had postoperative lymphatic fistula. However, following pressure dressing, fasting and other treatments, this complication was healed. However, incision separations were identified in 3 patients with the classic ' $L$ ' incision (Fig. 6).

One week after the surgery, all the patients were tested for their dermal sensations of the surgical region by acupuncture. Their dermal sensation function was staged. Among the 49 sides (47 cases in the cervical low incision group), 46 sides exhibited good dermal sensation, and the other 3 sides had no sensation at all. Among all the patients with cervical low incision, the surgical sides of 45 patients who received unilateral
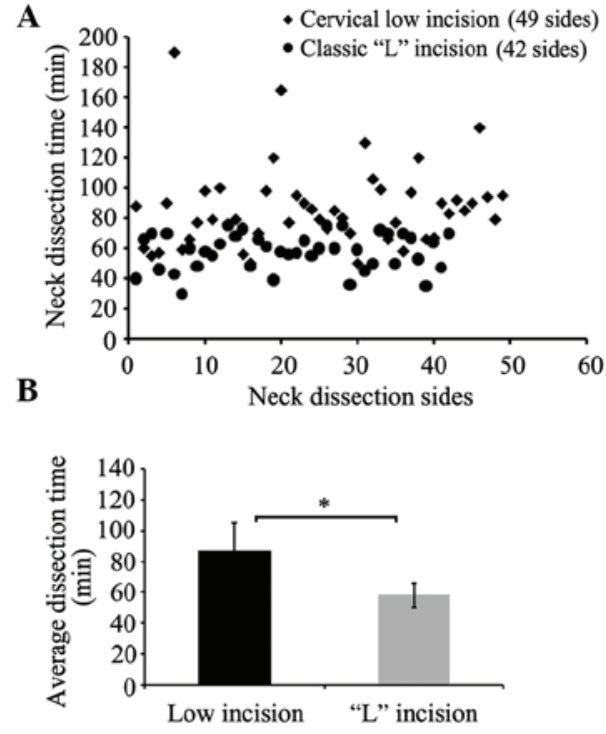

Figure 3. Neck dissection time. (A) Lateral neck dissection times of 47 patients (49 sides) with cervical low incision and 40 patients (42 sides) with classic ' $\mathrm{L}$ ' incision are shown in the scatter diagram. (B) The average neck dissection time of the cervical low incision group was compared with that of the classic ' $L$ ' incision group. ${ }^{*} \mathrm{P}<0.05$.
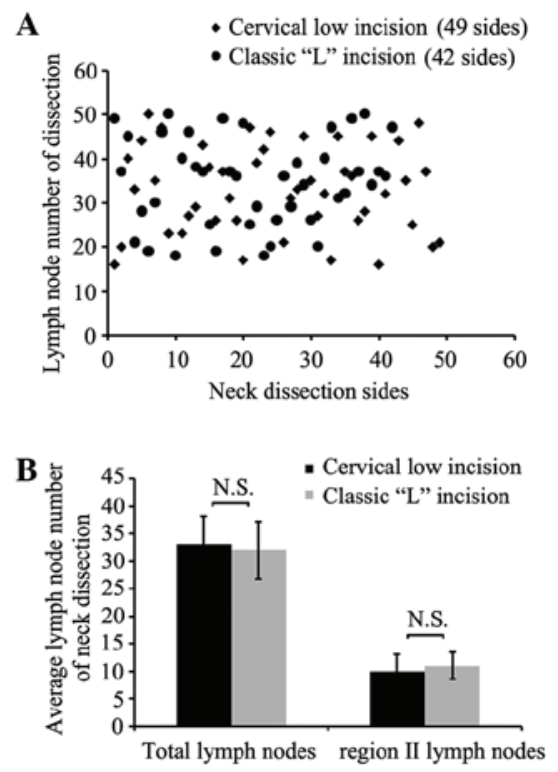

Figure 4. Lymph node numbers of neck dissection. (A) Lymph node numbers for 47 patients ( 49 sides) with cervical low incision and 40 patients ( 42 sides) with classic ' $L$ ' incision are shown in the scatter diagram. (B) The average total lymph node amount and the region II lymph node amount of unilateral neck dissection of the cervical low incision group were compared with that of the classic 'L' incision group. N.S., no significance.

neck dissection were tested for dermal sensation with respect to their contralateral normal skin. Among these 45 cases, 21 patients reported that the bilateral symmetry of cervical dermal sensations was similar, while the postoperative sides of 22 patients were slightly lower compared to the normal side, however, pains and tactual sensation were reported. The remaining 2 patients had no feeling on the postoperative sides. One patient in these 2 cases who underwent the bilateral 
Table III. Cervical lymph node metastasis.

\begin{tabular}{lccc}
\hline Variables & Total & Cervical low incision & Classic 'L' incision \\
\hline Cases, $\mathrm{n}$ & 87 & 47 & 40 \\
Dissection sides, $\mathrm{n}$ & 91 & 49 & 42 \\
Cases with lymph node metastasis, $\mathrm{n}(\%)$ & $65(74.7)$ & $35(74.5)$ & $30(75.0)$ \\
Dissection sides with lymph node metastasis, $\mathrm{n}(\%)$ & $69(75.8)$ & $37(75.5)$ & $32(76.2)$ \\
Metastasis node on each side, $\mathrm{n}$ & 4.8 & 4.2 & 0.6 \\
\hline
\end{tabular}

Table IV. Complication ratings.

\begin{tabular}{|c|c|c|c|c|c|c|}
\hline \multirow[b]{2}{*}{ Variables } & \multicolumn{3}{|c|}{ Cervical low incision } & \multicolumn{3}{|c|}{ Classic ' $L$ ' incision } \\
\hline & Total no. $(\%)$ & Unilateral & Bilateral & Total no. $(\%)$ & Unilateral & Bilateral \\
\hline Neck dissection cases, $\mathrm{n}$ & 47 & 45 & 2 & 40 & 38 & 2 \\
\hline Good dermal sensation, n (\%) & $22(46.8)$ & $21(44.7)$ & $1(2.1)$ & 0 & 0 & 0 \\
\hline Slightly weak sensation, n (\%) & $22(46.8)$ & $22(46.8)$ & 0 & 0 & 0 & 0 \\
\hline No dermal sensation, n (\%) & $3(6.4)$ & $2(4.3)$ & $1(2.1)$ & $40(100.0)$ & $38(95.5)$ & $2(5.0)$ \\
\hline Persistent neck pain, n (\%) & $4(8.5)$ & $4(8.5)$ & 0 & $11(27.5)$ & $10(25.0)$ & $1(2.5)$ \\
\hline Postoperative recurrence, $\mathrm{n}(\%)$ & $2(4.3)$ & $1(2.1)$ & $1(2.1)$ & $1(2.5)$ & $1(2.5)$ & 0 \\
\hline
\end{tabular}
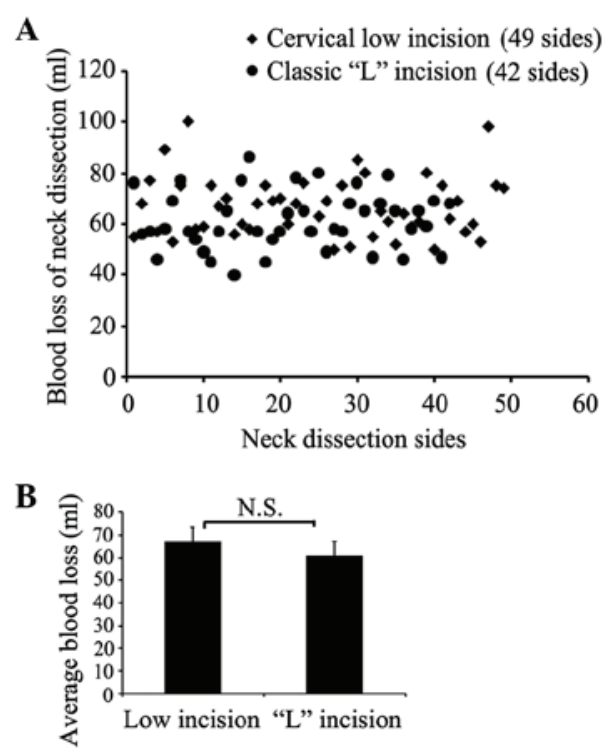

Figure 5. Blood loss during the neck dissection. (A) Blood losses of 47 patients (49 sides) with cervical low incision and 40 patients ( 42 sides) with classic ' $L$ ' incision are shown in the scatter diagram. (B) The average blood loss of the unilateral neck dissection of the cervical low incision group was compared with that of the classic 'L' incision group. N.S., no significance.

neck dissection had no pain and tactual sensation on 1 side (Table IV). However, among the 42 sides (40 cases in the classic 'L' group), all the sides nearly lost the feeling below the ear lobe and had persistent numbness of the ear lobe.

In addition, 4 of the 47 patients in the cervical low incision group had a persistent neck pain. However, this pain was tolerated and disappeared within 2 to 3 months after the surgery. There were 2 cases of cervical lymph node postoperative recurrence, 1 in the middle neck region (III area), and the other in the lower neck region (IV area). In the 2 cases, the cervical lymph nodes were excised. In total, 11 of the 40 patients in the classic ' $L$ ' incision group underwent a long and persistent neck pain, for almost 3 months (Table IV), resulting from the destruction of the fiber connection of the skin surface at the longitudinal incision and the large tension in the late healing process, which caused delayed healing (Fig. 6). There was 1 case of cervical lymph node postoperative recurrence in the IV area.

Postoperative satisfaction ratings. The neck scars of 43 patients (out of 47) in the cervical low incision group were not evident, as there was only a thin line on the lower neck (Fig. 7A). The other 4 patients had visible red scars, which were significant granulation tissues. However, the scars were located in the lower regions so that they could be covered (Fig. 7B). As the scars were lower than usual and could be covered by a collar, 25 patients reported satisfaction with this surgery. A total of 19 patients were primarily satisfied and only 3 patients were dissatisfied (Table V). However, the neck scars of almost all the patients in the classic ' $\mathrm{L}$ ' incision group were evident. The neck scars of 21 patients were an ' $L$ ' thin line extending from the lower neck to the ear (Fig. 8A), while the other 19 patients had clear red scars or granulations (Fig. 8B). However, as the scars were located in the exposed regions and could not be covered by a collar, 27 patients expressed dissatisfaction with this surgery. A total of 9 patients were primarily satisfied and only 4 were satisfied (Table V).

\section{Discussion}

The techniques of neck dissections have evolved from the radical neck dissection to present modified neck dissection, 
Table V. Postoperative satisfaction ratings.

\begin{tabular}{lcc}
\hline & $\begin{array}{c}\text { Cervical low } \\
\text { incision, } \mathrm{n}(\%) \\
(\mathrm{n}=47)\end{array}$ & $\begin{array}{c}\text { Classic ' } \mathrm{L} \text { ' } \\
\text { incision, } \mathrm{n}(\%) \\
(\mathrm{n}=40)\end{array}$ \\
\hline Evidiables & $4(8.5)$ & $40(100.0)$ \\
No evident neck scars & $43(91.5)$ & $0(0.0)$ \\
Satisfied & $25(53.2)$ & $4(10.0)$ \\
Basically satisfied & $19(40.4)$ & $9(22.5)$ \\
Dissatisfied & $3(6.4)$ & $27(67.5)$ \\
\hline
\end{tabular}

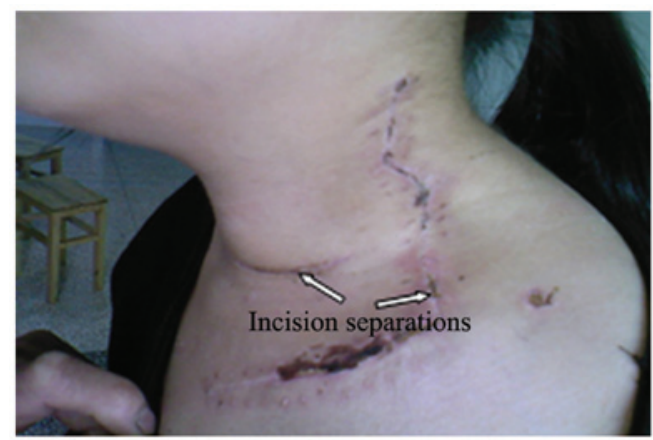

Figure 6. Incision separations are evident in the patients with the classic ' $\mathrm{L}$ ' incision.

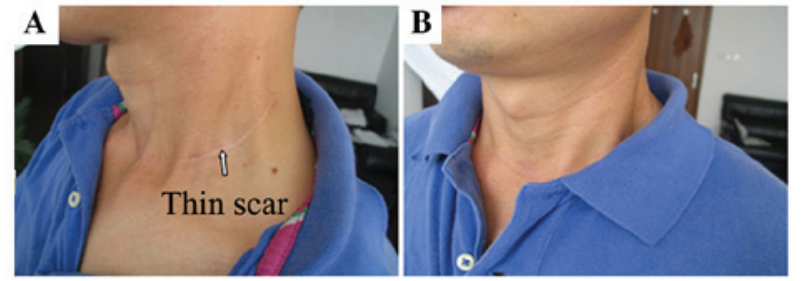

Figure 7. Neck appearance of patients following cervical low incision. (A) 3-year follow-up after surgery. (B) Scar was covered by a collar.

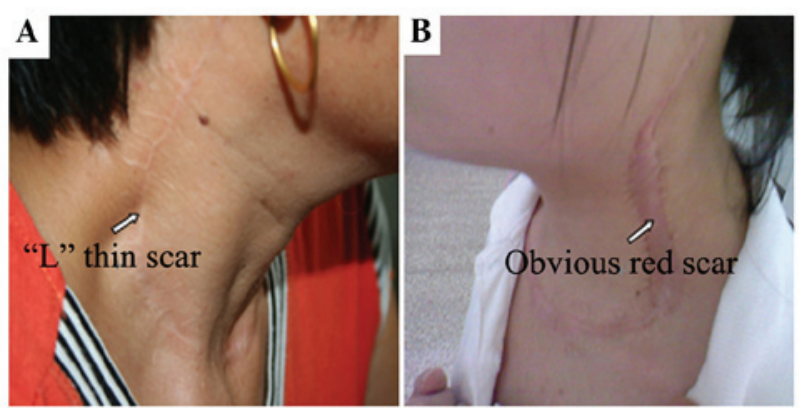

Figure 8. Neck scars of patients following the classic ' $L$ ' incision at the 3-year follow-up after surgery. (A) 'L' thin scar and (B) evident red scar.

which reserves the sternocleidomastoid, vena jugularis interna and accessory nerve. The conventional ' $L$ '-shaped incision has clear scars, particularly for the vertical section, which caused patient dissatisfaction regarding their appearance. The focus is currently on improving the patient quality of life. The
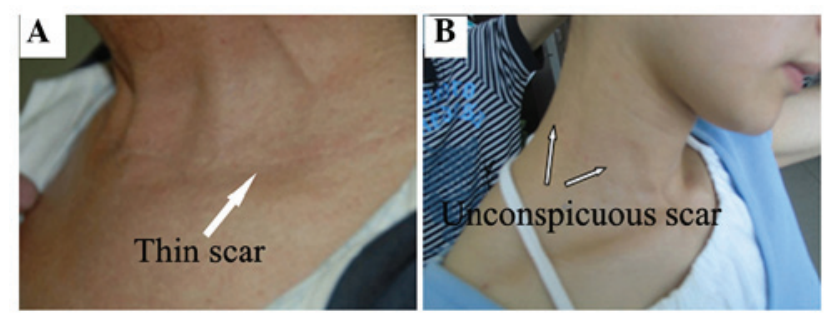

Figure 9. Inconspicuous thin neck scar of patients with cervical low incision at the 2.5-year follow-up after surgery. (A) Thin scar and (B) unconspicous scar.

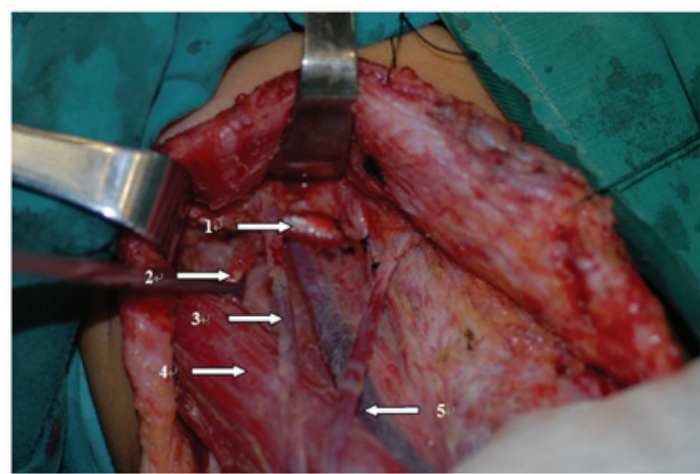

Figure 10. Good exposure of cervical low incision. 1., Digastric muscle; 2., accessory nerve; 3 ., vena jugularis externa; 4., sternocleidomastoid; 5. , vena jugularis interna.

modified neck dissection also has disadvantages. It requires the removal of the superclavicular and auricular nerves, and causes an inevitable result of loss of feeling below the ear lobe, including numbness, causing issues with patients, particularly for certain young patients. As thyroid papillary carcinoma has a favorable prognosis, maximizing the life quality of patients is important. Therefore, the low neck incision was applied to complete the neck dissection. Furthermore, the supracervical epithelial nerve, jugular vein and auricular nerve were conserved to reduce the impact of surgery on patients. This technique received satisfactory feedback.

Due to its good exposure, the conventional 'L'-shaped incision is accepted by the majority of surgeons. However, in the postoperative follow-up, due to the evident vertical section of the scar tissue, numerous patients expressed dissatisfaction. Therefore, the incision was first modified to be parallel to the neck dermatoglyph and $\geq 2 \mathrm{~cm}$ above the clavicle, making it a transverse incision that can cause a satisfactory appearance (Fig. 9A and B). In the follow-up of the 47 patients in the cervical low incision group, only 4 had an incision of granulation tissue hyperplasia with evident scars. The scars of the remaining 43 cases were inconspicuous. All these patients could cover their neck scars by their clothes (Fig. 7B). According to the relevant literature, Uchino et al (6) termed this an extended collar incision, Shah (7) defined it as the single transverse incision, Xi et al (8) termed it the long low collar incision and Zhang et al (9) termed it extending collar incision. These studies all agree that the incision scars are not evident and have less impact on the appearance.

With regards to the cleaning range, the range of conventional neck dissection was level II-VI lymph nodes and provided 
good exposure. At the beginning of the study, cervical low incision was not thought to provide as good an exposure to levels II and III as the conventional dissection. Therefore, it may obscure a thorough lymph dissection. However, in the present surgery, levels III and IV were well exposed. The exposure of level II was slightly worse compared to the conventional incision. However, once a full free flap was separated, an extremely good exposure to level II, as well as to level IIb, lymph nodes was obtained (Fig. 10). Therefore, the scope of the neck dissection was the same as the conventional neck dissection. When a patient required level I dissection, the surgery was more difficult due to the cervical low incision only. Therefore, in these types of cases, an auxiliary transverse neck incision (or MACFEE incision) is required to provide enough exposure. However, in the majority of papillary thyroid cancer cases, no exposure of level I is required, and the low incision can well replace the conventional neck dissection. Those patients, who have large cervical lymph nodes and dense adhesions following radiotherapy, may require conventional incision.

The patients who received routine lymph dissection always reported neck numbness or loss of the ability to feel pain around the ear lobe. Certain patients cannot sense when the skin of their neck is burned in that area. The loss of sensory function is due to the removal of the supracervical epithelial nerve and auricular nerve. For this reason, the neck dissection was modified by reserving the supracervical epithelial nerve and auricular nerve in addition to the conventional methods, which only reserves the sternocleidomastoid, vena jugularis interna and accessory nerve. This modification was successful (Fig. 2). Among the 49 sides (47 patients), 46 sides of the neck felt tactile pain. The preservation of sensory function was good. Postoperative sensory function of the neck was satisfactory. In theory, if the supracervical epithelial nerve and auricular nerve are retained, the patient should have a sensory function on the neck. However, there remain three sides of that neck that do not feel tactile pain, and 4 patients had severe neck pain. This may be due to nerve degeneration caused by the electric knife during skin flap separation. Therefore, how to preserve the nerve as well as its function requires further research. As for the surgery, based on the present experience, the neck has only to be exposed to two to three nerve roots to dissect level II and III lymph nodes from top to bottom and from the nerve cord to supracervical epithelial nerve branches. This feature makes the surgery less time consuming and more convenient.

Certain doctors are concerned that reserving so many normal structures may affect the outcome of neck dissection. However, the present results show that only 2 patients had cervical lymph node recurrence, which was only $4 \%$ of the sample. Those recurrences of lymph nodes were located in the lower neck, but not the poor exposure region (level II lymph nodes) of low incision. Therefore, we believe that good skill and full free skin flap can provide a good exposure, as well as the 'L'-shaped incision. Therefore, the effect of the cervical low incision neck dissection for 'eight reserved' (vena jugularis interna, vena jugularis externa, sternocleidomastoid, accessory nerve, superclavicular nerve, transverse cervical artery, musculus omohyoideus and auricular nerve) is not an issue. However, the long-term effect requires further follow-ups.

The reservation of vena jugularis externa can significantly reduce facial edema, while the omohyoid muscle is believed to have limited function. We believe that at the initial stage of treatment, these two can be sacrificed.

Due to poor exposure and fine dissection to retain a variety of normal tissues, 'eight reserved' cervical low incision neck dissection requires more surgical time compared to the conventional modified neck dissection.

Among the 47 cases in the cervical low incision group analyzed, only 2 had postoperative lymphatic fistula. No other complications were reported, indicating that the low incision neck dissection and 'eight reserved' did not increase the complications and were safe for patients. However, as low incision requires an upward pull flap to expose the surgical field, the surgery may cause tissue edema. However, it can be recovered quickly.

In conclusion, as living standards are improving, patients expect doctors to guarantee their qualities of life following surgery. The cervical low incision neck dissection for 'eight reserved' has only a hidden incision compared to the conventional method. Additionally, the scar tissue is not evident and the neck preserves sensation. These advantages meet the expectations of the patient. By contrast, it does not increase the suffering of patients and postoperative complications, since it conforms to eliminate the cervical lymph node and spare normal tissue requirements. Therefore, in the treatment of thyroid papillary carcinoma, low incision neck dissection for 'eight reserved' may replace the conventional modified neck dissection.

\section{Acknowledgements}

The present study was supported by the National 863 Fundamental Research Project (grant no. 2014AA02240), the National Natural Science Foundation of China (grant nos. 81550033 and 81202127), the Zhejiang Provincial Program for the Cultivation of High-Level Innovative Health Talents (grant no. 2008-134), and the Zhejiang Medical and Health Science and Technology Plan (grant nos. 2012KYA031, 2013KYB033, 2013KYB042 and 2014KYB038).

\section{References}

1. Shaha AR, Shah JP and Lorre TR: Patterns of nodal and distant metastasis based on histologic varities in differentiated carcinoma of thyroid. Am J Surg 172: 692-694, 1996.

2. Pisello F, Geraci G, Lo Nigro C, Li Volsi F, Modica G and Sciumè C: Neck node dissection in thyroid cancer. A review. G Chir 31: 112-118, 2010.

3. Palestini N, Borasi A, Cestino L, Freddi M, Odasso C and Robecchi A: Is central neck dissection a safe procedure in the treatment of papillary thyroid cancer? Our experience. Langenbecks Arch Surg 393: 693-698, 2008.

4. Daniel O and Robert U: Surgery of the thyroid and parathyroid glands. Berlin: Springer-Verlag, pp101-106, 2007.

5. Xue S, Wang P and Chen G: Neck dissection with cervical sensory preservation in thyroid cancer. Gland Surgery 2: 212-218, 2013.

6. Uchino S, Noguchi S, Yamashita H and Watanabe S: Modified radical neck dissection for differentiated thyroid cancer: Operative technique. World J Surg 28: 1199-2203, 2004.

7. Shah JP, Patel SG and Singh B: Head and neck surgery and oncology. Han DM, Yu ZK, translation. Philadelphia: Elsevier Mosby, p495, 2005.

8. Xi ZH, Wang QZ, Lu XB, et al: Long low collar incision in differentiated thyroid cancer radical surgery. Journal of Henan Medical University 37: 426-427, 2002.

9. Zhang B, Yan DG, An CM, et al: Application of an extended collar incision in neck dissection for differentiated thyroid cancer. Chin J Oncol 31: 223-225, 2009. 